Meta

Journal des tradlucteurs

Translators' Journal

\title{
La traduction poétique
}

\section{Albert Schneider}

Volume 23, numéro 1, mars 1978

La traduction poétique

URI : https://id.erudit.org/iderudit/004051ar

DOI : https://doi.org/10.7202/004051ar

Aller au sommaire du numéro

\section{Éditeur(s)}

Les Presses de l'Université de Montréal

ISSN

0026-0452 (imprimé)

1492-1421 (numérique)

Découvrir la revue

Citer cet article

Schneider, A. (1978). La traduction poétique. Meta, 23(1), 20-36.

https://doi.org/10.7202/004051ar

Ce document est protégé par la loi sur le droit d'auteur. L'utilisation des services d'Érudit (y compris la reproduction) est assujettie à sa politique d'utilisation que vous pouvez consulter en ligne.

https://apropos.erudit.org/fr/usagers/politique-dutilisation/
Cet article est diffusé et préservé par Érudit.

Érudit est un consortium interuniversitaire sans but lucratif composé de l'Université de Montréal, l'Université Laval et l'Université du Québec à Montréal. Il a pour mission la promotion et la valorisation de la recherche. https://www.erudit.org/fr/ 
gear $\gg$, the vulnerable in-between moment. There may be no exact cultural equivalent - indeed the whole custom of « unwinding 》 may be peculiar to « commuting societies» - but I should have thought that the cocktail lounge in the U.S.A., the pavement café in France, and the Kaffeehaus or Konditorei in Germany and Austria would be much closer equivalents in terms of atmosphere and psychological function.

A warning in conclusion. The translation of poetry, as every practitioner knows, is beset by dozens of problems, pitfalls, hurdles and obstacles. Some of these are generic, in the sense that they recur in some form or other, while others are specific to a particular text. Of the generic ones I have, in this article, discussed only two : (1) alternatives, (2) metaphor. But I have no doubt that these two are responsible for more translation failures than all other difficulties combined.

I believe that the nature of the translation problems can be analyzed and defined, and that a general strategy for tackling them - or, at least, the generic ones - can be mapped out. But I do not believe that anything like a detailed tactic, blueprint or drill - one that would be applicable to all situations - can ever be developed. Within a broad general strategy specific solutions must always remain intuitive.

EWALd Osers 


\section{La traduction poétique*}

Les réflexions qui suivent ne sont pas d'un théoricien, mais d'un praticien. Celui-ci ne dédaigne nullement la théorie. Mais dans un tel domaine, celle-ci est peu de chose sans la pratique qui, conjointement avec l'observation, c'està-dire avec la lecture et l'appréciation de traductions poétiques nombreuses et diverses, fait découvrir les vrais problèmes et conduit à un certain nombre de constatations dont plusieurs peuvent être érigées en règles. Celles-ci pourront être accueillies, discutées, justifiées ou contestées par les théoriciens. Le traducteur de poésies aura tendance, peut-être à tort, à s'en tenir aux règles qu'il a luimême découvertes.

Comme tout le monde, l'auteur de cet article a fait bien des traductions dans sa vie : il a traduit du latin, du grec, de l'allemand, un peu d'anglais, de suédois et d'italien. Comme tout le monde, il a commencé par la prose. Et lorsqu'il avait à traduire des vers, il les traduisait en prose en essayant d'éviter la platitude et le ridicule. Comme tous les universitaires, il cherchait avant tout l'exactitude et, autant que possible, l'élégance. Dans les collections bilingues, on rencontre beaucoup de traductions de ce genre-là. Elles sont en général fidèles, parfois agréables à lire, rarement brillantes. La plupart sentent la traduction et, lorsqu'il s'agit de poésie, elles sont franchement décevantes. Utiles et exactes, elles n'ont rien d'artistique ou de poétique. Sans le texte original, elles seraient illisibles, insupportables.

Il est assez naturel qu'un lecteur exigeant, doué de sens littéraire et poétique, ne puisse à la longue se contenter de ces traductions sérieuses et honorables, mais qui transforment une princesse en Cendrillon. C'est l'aventure qui est arrivée à l'auteur de ces pages et qui l'a poussé à s'attaquer à l'une des tâches les plus difficultueuses qui soient et à s'imposer d'innombrables « exercices $\gg$ - au sens valéryen du mot ${ }^{1}$.

* Pour ne pas allonger démesurément cet article, l'auteur renonce à donner une bibliographie. On en trouvera quelques éléments dans les notes.

1. $\mathrm{P}$. Valéry, "Propos me concernant $»$, in : Berne-Joffroy, Présence de Valéry, Ed. Raoul Henry, Bruxelles, Plon, Paris, p. 51: «Je regarde la poésie comme le genre le moins idolâtre. Elle est le sport des hommes insensibles aux valeurs fiduciaires du langage commun... Je viens de prononcer le mot sport. C'est que je rapporte tout ce que je pense de l'art à l'idée d'exercice, que je trouve la plus belle idée du monde... C'est le grand art pour moi que de dresser l'animal Langage et de le mener où il n'a pas coutume d'aller; mais de l'y mener avec l'apparence de la liberté la plus dégagée.... 
Plusieurs maîtres ont marqué mes débuts de traducteur de poésie. Je ne citerai que Maurice Boucher, professeur à la Sorbonne, directeur des Etudes germaniques. C'était un grand germaniste, mais aussi un artiste. Il était l'auteur de plusieurs recueils de poèmes. En 1941, il publia un Choix de poèmes de Stefan George, suivi d'un $2^{\mathrm{e}}$ volume en $1943^{2}$. Quand je lus ces traductions poétiques, je fus stupéfait et restai confondu d'admiration devant leur perfection. Maurice Boucher respectait toutes les règles que les universitaires observent généralement dans leurs traductions : il n'omettait rien, n'ajoutait rien, ne déformait rien. Mais, en plus, avec un goût très sûr et un immense talent poétique, il transposait aussi l'atmosphère des poèmes de Stefan George, leur rythme, leur musique. Donnons-en un court exemple.

\section{Jahrestag}

O schwester nimm den krug aus grauem thon

Begleite mich ! denn du vergassest nicht

Was wir in frommer wiederholung pflegten.

Heut sind es sieben sommer dass wirs hörten

Als wir am brunnen schöpfend uns besprachen :

Uns starb am selben tag der bräutigam.

Wir wollen an der quelle wo zwei pappeln

Mit einer fichte in den wiesen stehn

Im krug aus grauem thone wasser holen.

\section{Anniversaire}

Viens, ma sœur, suis-moi, prends l'amphore de grès,

Allons ensemble, c'est le jour, il t'en souvient,

Le rite est entre nous pieusement gardé.

C'est le septième été que là-bas nous apprîmes

Tandis que nous causions en puisant à la source

La mort, le même jour, de nos deux fiancés.

Allons à la fontaine où les deux peupliers

Se dressent dans les champs près de l'épicea

Nous puiserons de l'eau dans l'amphore de grès.

(Maurice Boucher ${ }^{3}$ )

On constate qu'avec des moyens différents le traducteur sait produire la même impression que le poète allemand. Sa transposition est fidèle, mais sans servilité. C'est un travail d'artiste, et non d'universitaire.

Maurice Boucher a aussi traduit en vers deux pièces de thêâtre de Goethe : Iphigénie et Le Tasse. Ce sont les pièces classiques de Goethe, celles qui par leur style pur, limpide, noble et leur musique cristalline rappellent étrangement les pièces de Racine. $M$. Boucher les a transposées dans des vers qui ne sont indignes ni de Racine, ni de Goethe. Sa réussite est parfaite, insurpassable. Citons les 14 premiers vers d'Iphigénie, puis la traduction de M. Boucher.

Heraus in eure Schatten, rege Wipfel

Des alten, heil'gen, dichtbelaubten Haines,

Wie in der Göttin stilles Heiligtum,

2. Ed. Aubier, Paris, 2 vol., 1941 et 1943. Réed. chez Aubier-Flammarion, Paris, 1969

(en 1 vol.)

3. S. George, Dichtungen. Poèmes, Aubier-Flammarion, 1969, p. 80, 81. 
Tret' ich noch jetzt mit schauderndem Gefühl,

Als wenn ich sie zum erstenmal beträte,

Und es gewöhnt sich nicht mein Geist hierher.

So manches Jahr bewahrt mich hier verborgen

Ein hoher Wille, dem ich mich ergebe ;

Doch bin ich, wie im ersten fremd.

Denn ach ! mich trennt das Meer von den Geliebten,

Und an dem Ufer steh' ich lange Tage,

Das Land der Griechen mit der Seele suchend;

Und gegen meine Seufzer bringt die Welle

Nur dumpfe Töne brausend mir herüber.

(Goethe)

Quand je viens sous votre ombre, ô cimes frémissantes

Dans le feuillage dru de vos arbres sacrés

Comme au silence auguste où règne la déesse

Je ne puis maîtriser un effroi dans mon coeur :

Un frisson me saisit comme le premier jour

Mon âme parmi vous ressent toujours l'exil.

Les ans ont fui, depuis que me cache en ces lieux

La volonté du ciel qui me trouve soumise.

Pourtant comme autrefois je me sens étrangère :

Ceux que j'aimais sont loin, loin par delà les mers,

Je reste de longs jours, debout sur le rivage

Et la pensée errante, au pays des Hellènes.

En réponse à ma plainte il ne vient de là-bas

Que le sourd bruissement des vagues mugissantes.

(Maurice Boucher ?)

Une telle perfection est décourageante. L'admiration peut être paralysante. Aussi n'ai-je pas, en ces années, essayé d'imiter les prouesses de mon maître.

C'est l'insatisfaction qui, chez moi, s'est faite créatrice. Dans différentes publications, il m'arrivait de lire des traductions de poésies allemandes. Je les trouvais souvent bien mauvaises. Et quand il s'agissait de poèmes que j'aimais, j'en souffrais. C'est ainsi que je me suis mis à transposer des poèmes - en essayant de faire mieux que mes prédécesseurs, mes anti-modèles. Mon idéal était de fournir des traductions à la fois fidèles et poétiques. Je ne tardai pas à m'apercevoir que c'était vouloir réaliser la quadrature du cercle. Mais fortifié par l'exemple de mon maître Maurice Boucher et de certains autres traducteurs, je ne me suis pas laissé décourager par la masse des difficultés de toutes sortes qui surgissent au cours du travail de transposition. Je me redisais parfois les vers de Paul Valéry :

Patience, patience,

Patience dans l'azur!

Chaque atome de silence

Est la chance d'un fruit mûr ${ }^{5}$ !

Vers que R.M. Rilke, qui a traduit pas mal de Valéry, transpose de la façon suivante :

4. Goethe, Iphigénie en Tauride, traduction de Maurice Boucher, Ed. Stock, Paris, 1943,

5. p. Valéry, Euvres, Bibliothèque de la Pléiade, Gallimard, 1957, t. I, p. 155. 
Gedulden, Gedulden, Gedulden,

Gedulden unter dem Blau!

Was wir dem Schweigen verschulden,

Macht uns das Reifen genau ${ }^{6}$.

Traduction que je trouve, sauf le respect que je dois à Rilke et que je lui porte réel'ement, décevante : elle empâte les vers de Valéry et s'écarte du texte, qu'elle obscurcit, en grande partie parce que Rilke a voulu rimer. Mais laissons cette question, nous y reviendrons plus tard.

Et revenons aux raisons qui m'ont poussé à traduire des poèmes. J'ai donc transposé certains poèmes parce que le hasard avait mis sous mes yeux une traduction qui me paraissait inadéquate, trop libre ou trop littérale, trop inexacte ou trop peu poétique. J'en ai traduit d'autres parce que le thème m'intéressait (par exemple Venise); d'autres à cause de la manière du poète, de sa technique ; d'autres parce que je connaissais personnellement l'auteur et voulais lui faire plaisir et lui faire l'hommage d'une traduction soignée; d'autres parce que leur atmosphère me plaisait et que je me sentais des affinités avec l'auteur; d'autres enfin parce qu'ils me paraissaient très difficiles à traduire et qu'ils suscitaient en moi l'irrésistible envie de vaincre la difficulté, un peu comme un alpiniste choisit de gravir la pente la plus raide et la plus dangereuse. Je pratique donc, par périodes, une sorte d'alpinisme poétique qui me permet de respirer, au sortir du marécage pédagogique et universitaire, un air plus pur et plus vif et de retrouver par là équilibre intellectuel et santé morale. Quelle satisfaction de tenter - et parfois de réussir - l'impossible! Disons enfin que je ne ferais pas de traductions poétiques si je n'aimais pas la poésie, qui est pour moi à la fois un paradis perdu et une terre promise.

Mes lectures et mon expérience m'ont permis de faire un ensemble de constatations, d'observations, de remarques qui m'ont conduit à formuler quelques règles, à statuer certains principes - pour moi plus que pour les autres. Il est bien entendu que tout cela demeure empirique du fait que la traduction poétique est, comme la médecine, à la fois une science et un art.

Dans la traduction poétique en général, il convient tout d'abord de distinguer des catégories. Là, je suis d'accord avec deux traducteurs allemands très estimés : Karl Dedecius et Friedhelm Kemp, dont le premier est un slavisant et l'autre un romaniste. Je distingue, avec eux :

1) la traduction simple, courante ;

2) la traduction artistique et poétique, que je nommerais volontiers «transposition » ou, reprenant le vieux mot français utilisé par Ronsard et Du Bellay, «translation ».

Dedecius et Kemp sont d'accord sur le fond, mais non sur la terminologie, qui est assez confuse. Dedecius distingue :

a) die Übersetzung, qu'il dit «zuverlässig, aber unkünstlerisch », ce qui est une bonne définition;

6. P. Valéry, Gedichte, Französisch und deutsch, Übertragen durch R.M. Rilke, Rowohlt, rororo, p. 63. 
b) die Übertragung, qui est selon lui « künstlerisch und zuverlässig »;

c) die Nachdichtung, qu'il dit «künstlerisch, aber unzuverlässig ». La définition est bonne, mais le terme de Nachdich est impropre; on verra que c'est Umdichtung qui convient?

Friedhelm Kemp distingue à son tour :

a) die Übertragung qu'il définit « eine mehr oder minder interpretierende Interlinearversion, die um der Wörtlichkeit willen manches von der Form opfert und auf eine strenge Beobachtung des Versmasses, des Reimschemas verzichtet», c'est-à-dire une traduction d'un niveau modeste, sans ambition, sans envergure; à mon avis, le terme de Übertragung, plus noble que Übersetzung, est mal choisi ;

b) die Nachdichtung, « jene Art der Herübernahme..., welche die äussere Form der Vorlage um jeden Preis zu retten sucht, selbst auf Kosten alles dessen, was die Schönheit, Kraft und Originalität des Gedichtes ausmacht », définition plutôt imparfaite et assez défavorable, semble-t-il ; contredite d'ailleurs par un autre texte de Kemp, plus juste et plus compréhensif : "Die Nachdichtung gehört seit jeher [...] zum Handwerk des Dichters. Verse eines anderen Dichters haben es ihm angetan; Liebe und Bewunderung wecken das Verlangen nach innigerem Besitz und zugleich den Wunsch, das fremde Schöne in der eigenen Sprache einzubürgern... Der Dichter nimmt das fremde Werk gleichsam in sich hinein, steigt mit ihm auf jenen Grund hinab, wo Sprache nicht schon dieses oder jenes geschicht lich Gewordene und Differenzierte ist, wo sie noch als reine Möglichkeit alles Sagens braut und west; von dort hofft er, zu günstiger Stunde, mit dem ebenbürtigen Gedicht in der eigenen Mundart heraufzukommen »;

c) die Umdichtung, qu'il définit « die mehr oder minder freie Verwendung, Umgestaltung, ja Auflösung des Originals zu eigenen, neuen Zwecken ${ }^{8}$. 》

Personnellement, je serais partisan (en allemand) de la terminologie suivante :

a) Übersetzung pour la traduction courante, exacte, mais sans grande prétention, sans ambition artistique;

b) Übertragung ou mieux, Nachdichtung pour la traduction poétique, mais fidèle ;

c) Umdichtung pour une transposition brillante, mais infidèle, trop personnelle.

Bien entendu, les frontières entre ces trois genres de traduction sont flottantes. Il y a des traductions sans prétention, mais soigneuses qui se rapprochent d'une Übertragung parce qu'elles présentent des qualités littéraires, voire poétiques. Il y a des traductions poétiques qui hésitent entre la Nachdichtung et la Umdichtung. C'est dans ce domaine que je rangerai les traductions que Rilke a faites de Valéry et que George a faites de Baudelaire. Rilke et George étaient de trop grands poètes pour être vraiment fidèles aux auteurs traduits. Leurs

7. Karl Dedecius, Slawische Lyrik, übersetzt, übertragen, nachgedichtet, in : Das Problem des Übersetzens, Wissenschaftliche Buchgesellschaft, Darmstadt, 1963, p. 469.

8. Friedhelm Kemp, Kunst und Vergnügen des Übersetzens, Neske, Pfullingen 1965, p. $16,17,18$. 
transpositions sont du Rilke et du George autant que du Valéry et du Baudelaire. Nous y reviendrons.

On peut traduire un poème de bien des façons.

1) Tout d'abord littéralement. C'est la version interlinéaire qu'on a beaucoup pratiquée au Moyen-Âge et qui s'est révélée précieuse pour les linguistes et les historiens des langues à qui elle apporte une foule de renseignements. Cette sorte de traduction reprend à l'heure actuelle une certaine importance. En effet, beaucoup de poètes du $\mathrm{xx}^{\mathrm{e}}$ siècle sont obscurs, voire hermétiques, difficiles à interpréter. La meilleure façon de les traduire est donc de les traduire assez littéralement. Une traduction n'est pas une interprétation, un commentaire. Si le poème original est obscur, la traduction doit l'être aussi. Cette règle n'est pas aussi facile à observer qu'on le pense. Il y a une question de langue qui joue. Le français est une langue plus claire, plus nette que l'allemand. De ce fait, la traduction française sera presque toujours plus claire - ou moins obscure que l'original allemand. C'est une observation qui m'a été faite par des lecteurs allemands de mes transpositions. Ils se sont rendu compte que le traducteur était probablement moins responsable de cette clarification que la langue elle-même, c'est-à-dire le français.

2) On peut encore traduire des vers en prose soignée, voire poétique. C'est la solution sage et prudente. Elle permet d'être à la fois exact et élégant. Mais elle sacrifie le rythme et la musique du vers, c'est-à-dire un élément essentiel du poème, sinon l'élément essentiel. C'est le genre de traduction que pratiquent la plupart des universitaires et qui font le bonheur des éditeurs de publications bilingues, des étudiants de langues et des personnes qui sont incapables de lire les auteurs étrangers dans le texte. Il est très légitime, très utile, je dirai même indispensable. Mais généralement, la traduction en prose ne donne pas de grandes satisfactions artistiques. Je dis généralement, car il y a des exceptions. Certains universitaires fournissent des traductions d'un niveau élevé. Quelques poètes n'ont pas craint de traduire des vers en prose, par exemple Baudelaire et Mallarmé. Tous deux ont traduit en prose le célèbre poème du Corbeau, d'Edgar Allan Poë. Friedhelm Kemp trouve la version de Baudelaire excellente, mais celle de Mallarmé encore meilleure : «Mallarmé aber, der ein immer waches Gefühl für alle Möglichkeiten syntaktischer Gliederung und Brechung der Prosa besass, schafft in seiner Übertragung durch kleine Kunstgriffe der Inversion, der Weglassung von Artikel, Personalpronomen und Konjunktion allerlei Einschnitte und Verstrebungen, die als ein gültiger Ersatz für Versmass und Strophengliederung wirken ${ }^{\circ}$. $>$ Personnellement, je pense qu'une telle traduction n'est tout de même pas l'idéal : elle laisse perdre encore bien des choses; elle ne donne plus vraiment l'impression d'un poème. Citons d'autres exemples. Wilhelm Heinse, l'auteur d'Ardighello, a traduit en prose les stances de La Gerusalemme liberata du Tasse. F. Kemp lui-même a fourni des Fleurs du mal de Baudelaire une traduction en prose ${ }^{10}$. Une tentative lucide et qui en valait la peine parce

9. F. Kemp, Kunst und Vergnügen, p. 23, 24.

10. Charles Baudelaire, Die Blumen des Bösen, Aus dem Französischen übertragen von F. Kemp. Fischer-Bücherei Nr. 737. 
que Stefan George, qui a traduit en vers le même recueil, avait surtout vu en Baudelaire la rigueur parnassienne et «l'ardente spiritualité »; il avait donc donné à ses transpositions des Fleurs du mal noblesse et dignité, négligeant d'autres aspects de l'œuvre et de la personnalité : en particulier le réalisme du poète et son modernisme. Voici dans quel esprit Kemp a conçu sa traduction : "Diese Skizze sollte nach grösster Treue des Sinnes trachten; jeder Gedanke so!lte seine Schärfe, jedes Bild seine sinnliche Deutlichkeit behalten; nichts sollte verbogen, nichts geschönt oder mit gefühliger Stimmung verklebt werden. Glanz und Musik des Originals freilich waren so nicht zu retten; aber vielleicht war es möglich, dem deutschen Leser zum ersten Male die Hilflosigkeit, die Ausgesetztheit dieses Dichters zu zeigen, seine Verlorenheit, aus der er den unerhörten Ton gewinnt, die gebrochene Stimme, die das Pathos des klassischen Alexandriners unterhöhlt und zersetzt ${ }^{11}$. $\gg$ Une traduction en prose très soignée peut donc avoir sa justification et faire apparaître des choses qu'une Nachdichtung risque de masquer ou de voiler.

3) On peut traduire un poème en vers réguliers ou libres, rimés ou non rimés. C'est là qu'on peut parler de Nachdichtung, de re-création, de transposition.

La traduction poétique doit être à la fois fidèle et belle, c'est-à-dire poétique. On ne doit rien ajouter, rien retrancher au texte original. Bien entendu, il ne saurait s'agir d'une fidélité littérale, minutieuse et pour ainsi dire anxieuse. On peut parfois condenser, laisser tomber un mot peu important; on peut aussi, parfois, expliciter - en veillant à ne pas délayer. Il s'agit de produire, dans la langue cible, un effet qui soit comparable à l'effet produit par le texte original, mais avec d'autres moyens. Il faut que la transposition (j'aime ce terme emprunté aux musiciens) soit digne d'être lue pour elle-même, sans recours au texte primitif. En dépit des apparentes libertés qu'elle prend (par ex. remplacer un verbe par un substantif, un adjectif par un nom, etc.) la transposition est souvent plus fidèle que la traduction littérale. Mais il s'agit de fidélité globale et de l'effet d'ensemble, le seul qui compte finalement.

Les traductions poétiques faites par des poètes sont rarement très fidèles. Nous avons évoqué tout à l'heure les transpositions faites par Rilke de Valéry et par Stefan George de Baudelaire. Outre qu'on relève dans ces textes des erreurs de sens dues à une connaissance insuffisante du français ou de la pensée ou de la personnalité des auteurs français, le lecteur constate que les transpositions en question sont plus proches de la manière de Rilke et de George que de celle de Valéry et de Baudelaire. Donnons-en de courts exemples. Dans les Fleurs du mal, deux poèmes sont intitulés Femmes damnées. Le deuxième commence par la strophe suivante :

Comme un bétail pensif, sur le sable couchées

Elles tournent leurs yeux vers l'horizon des mers,

Et leurs pieds se cherchant et leurs mains rapprochées

Ont de douces langueurs et des frissons amers.

11. F. Kemp, Kunst und Vergnügen, p. 24. 
George a traduit cette strophe de la manière suivante :

Wie rinder sinnend auf den uferkieseln

So blicken sie zum fernen himmelsrand,

Mit sanftem sehnen und mit fieberrieseln

Verschlingt sich fuss mit fuss und hand mit hand.

C'est incontestablement une belle strophe et, qui plus est, rimée. Mais est-elle fidèle? George a rendu l'alexandrin, qui est un vers majestueux, par le vers iambique à cinq pieds, donc par un décasyllabe, qui est plus léger, mais correspond mieux aux habitudes allemandes. Si les tragédies françaises sont écrites en alexandrins, les drames allemands sont écrits, depuis le dernier tiers du xvIIr siècle, en vers ïambiques à 5 pieds. D'une certaine façon, les vers se correspondent donc. Mais George a retranché et rajouté des choses : uferkieseln ne correspond pas à sable, l'horizon des mers n'est pas tout à fait traduit par zum fernen himmelsrand (il manque la mer, élément important) ; verschlingt sich fuss mit fuss est trop précis pour leurs pieds se cherchant; rinder est d'un niveau stylistique supérieur à bétail ; auf den uferkieseln se rattache grammaticalement à Wie rinder sinnend alors que sur le sable couchées se rapporte aux femmes et non au bétail. Le mot mit quatre fois répété dans les vers 3 et 4 est un peu gênant. Quant à la musique des voyelles, il y a dans la strophe de George trop de i (15!); le texte français, qui n'en comporte que 4 , a une tonalité plus sombre. Ici, la Nachdichtung frôle dangereusement la Undichtung. Pour bien s'en rendre compte, il suffit de lire la traduction en prose de F. Kemp :

Wie Vieh, nachdenkliches, so ruhn sie auf dem Sande und wenden ihre Augen nach dem Horizont der Meere ;

und ihre Füsse suchen, ihre Hände greifen sich

in sössem Sehnen und mit bitterem Schaudern ${ }^{12}$.

Passons à Rilke. Voici la dernière strophe de la Pythie, de Paul Valéry:

Honneur des hommes, Saint Langage,

Discours prophétique et paré,

Belles chaînes en qui s'engage

Le dieu dans la chair égaré,

Illumination, largesse!

Voici parler une Sagesse

Et sonner cette auguste Voix

Qui se connaît quand elle sonne

N'être plus la voix de personne

Tant que des ondes et des bois !

Rilke, avec beaucoup de talent, en a fait ceci :

Der Menschen Stolz, Sprache der Gnaden, die festlich ins Künftige sieht

und den Gott, wie an köstlichem Faden,

durch die Wirrsal des Fleisches zieht,

Erleuchtung, Verklärung, Verschwendung!

Hier spricht eine weise Vollendung, und eine Stimme erschallt,

12. Citations d'après F. Kemp, Kunst und Vergnügen, p. 25. 
die klingt, als ob sie erkläre,

dass sie niemandes Stimme mehr wäre,

so gehört sie zu Wasser und Wald ${ }^{13}$.

Les inexactitudes et déformations sont nombreuses. Stolz pour honneur peut passer. Mais Sprache der Gnaden pour Saint Langage ne va pas du tout. Le $2^{\mathrm{B}}$ vers : die festlich ins Künftige sicht rend mal Discours prophétique et paré - sans compter l'incohérence de l'image : Die Sprache, die ins Künftige sieht (image qui n'est pas dans le texte de Valéry). Belles chaînes en qui s'engage / Le dieu dans la chair égarê a été encore plus mal rendu par und den Gott, wie an köstlichem Faden / durch die Wirrsal des Fleisches zieht.

Le sens (les belles chaînes sont celles de la poésie, de la versification) ne semble pas avoir été saisi ; chaînes est rendu par Faden qui produit ici un effet un peu ridicule. Die Wirrsal des Fleisches semble rendre dans la chair égaré; mais Rilke ne s'est pas rendu compte qu'il y a une inversion pour signifier : le dieu égaré dans la chair. L'image est pénible : die Sprache zieht den Gott an köstlichem Faden durch die Wirrsal des Fleisches; elle n'est pas non plus dans le texte de Valéry. Dans le vers suivant, illumination est traduit doublement par Erleuchtung, Verklärung. En dehors de l'erreur de sens due à une mauvaise connaissance des idées de Valéry, la rime est en grande partie responsable de la catastrophe : Faden a été amené par Gnaden et sieht est là pour rimer avec zieht. Là aussi, nous frôlons la Umdichtung, l'adaptation.

Justement, il nous faut dire ici un mot de la Umdichtung. C'est un genre qui a été beaucoup pratiqué au Moyen-Âge, au XVI ${ }^{\mathrm{e}}$, au $\mathrm{XVII}^{\mathrm{e}}$ et au XVIII ${ }^{\mathrm{e}}$ siècle. Les poètes tant allemands que français s'inspiraient d'un passage de la Bible, par ex. d'un psaume, pour écrire de très beaux poèmes. C'était ce qu'on appelait «paraphrase» et ce que j'appellerais volontiers d'un terme musical une « variation » sur un thème donné. Si le principe de la liberté du "paraphraseur» est admis, le genre, où l'on compte de nombreuses et belles réussites, est parfaitement légitime ${ }^{14}$.

Il n'a pas entièrement disparu. Il y a encore des poètes qui, séduits ou émus par un poème, éprouvent l'envie de le traduire, mais très librement, conformément à leur tempérament, à leur manière. Ils le recréent et en font quelque chose de très beau, mais de très différent de l'original. On en donnera un exemple tiré de Verlaine, un poète qui a inspiré nombre de traducteurs. Richard Dehmel a transposé la Lune blanche, un très beau morceau du recueil la Bonne Chanson.

La lune blanche

Luit dans les bois;

De chaque branche

Part une voix

Sous la ramée...

$\mathrm{O}$ bien-aimée.

13. P. Valéry, Gedichte, Übertragen durch R.M. Rilke, rororo, p. 38, 39.

14. F. Kemp, «Vom Übersetzen französischer Dichtung $»$, in : F. Kemp, Kunst und Vergnügen, p. 19. 
L'étang reflète

Profond miroir,

La silhouette

Du saule noir

Où le vent pleure...

Rêvons, c'est l'heure.

Un vaste et tendre

Apaisement

Semble descendre

$\mathrm{Du}$ firmament

Que l'astre irise...

C'est l'heure exquise.

Paul Verlaine ${ }^{15}$

Ce poème est composé de vers très courts, très musicaux, d'une part à cause des rimes, d'autre part à cause de la musique très raffinée des voyelles et des consonnes. C'est une gageure de vouloir traduire un tel poème, dont presque tout le charme réside dans la forme. Dehmel a bien senti ce charme et n'a pu résister au désir de tenter une semblable incantation dans sa propre langue. Voici sa transposition, qu'on hésite à appeler traduction :

Helle Nacht

Weich küsst die Zweige

der weisse Mond;

ein Flüstern wohnt

im Laub, als neige

als schweige sich der Hain zur Ruh,

Geliebte du.

Der Weiher ruht, und

die Weide schimmert;

ihr Schatten flimmert

in seiner Flut, und

der Wind weint in den Bäumen,

Wir träumen - träumen.

Die Weiten leuchten

Beruhigung,

Die Niederung

hebt bleich den feuchten

Schleier hin zum Himmelssaum.

$\mathrm{O}$ hin - o Traum.

Richard Dehmel ${ }^{16}$

Tout d'abord, on notera que le traducteur a su, lui aussi, jouer admirablement de la musique des voyelles et des consonnes. Son poème a la même douceur

15. Paul Verlaine, Euvres poétiques complètes, Bibliothèque de la Pléiade, Gallimard, Paris, 1951, p. 105, 106.

16. La traduction de R. Dehmel est citée par W.E. Süskind et Georg von der Vring dans un article-dialogue intitulé : «Die Kunst der Übersetzung», article recueilli dans un ouvrage collectif intitulé lui-même Die Kunst des Übersetzung. Vorträge, Aufsätze, Hrsg. von der Bayerischen Akademie der schönen Künste, Verlag R. Oldenbourg, München, 1963, p. 17. 
rêveuse et sentimentale que celui de Verlaine. Mais Dehmel a pris bien des libertés. Il respecte la strophe de 6 vers, mais non le caractère des vers. Les siens sont irréguliers ( 2 et 3 accents), alors que ceux de Verlaine sont très réguliers (4 syllabes). Il s'écarte beaucoup du texte : Weich küsst die Zweige est surajouté, d'ailleurs un peu doucereux. Als neige / als schweige sich der Hain zur Ruh déforme gravement le sens. Verlaine dit qu'une voix part de la ramée, et non qu'elle se tait! C'est sans doute cette voix qui dit : $O$ bien-aimée:

Passons tout de suite à la $3^{\text {e }}$ strophe. Celle de Dehmel n'a pas du tout le charme mystérieux de celle de Verlaine. Chez celui-ci, il y a un mouvement de descente; chez Dehmel, c'est une monté, et triste, voire sinistre : die Niederung / hebt bleich den feuchten / Schleier hin zum Himmelssaum. C'est le dernier vers qui est le plus infidèle, non seulement dans la lettre, mais dans l'esprit : $O$ hin - $o$ Traum. C'est un vers qui marque la fin d'un rêve, qui constitue un dénouement mélancolique. Alors que chez Verlaine nous sommes en plein dans un rêve tendre et heureux : C'est l'heure exquise. La transposition de Dehmel est l'œuvre d'un grand poète. Mais du poème rêveur et léger de Verlaine il a fait quelque chose de triste, de mélancolique, bref de très germanique. La $3^{\mathrm{e}}$ strophe de Dehmel n'est pas sans rappeler maintes strophes de Volkslied ou de ballade. C'est une toute autre atmosphère que l'atmosphère verlainienne.

Comme on le voit, les grands poètes sont assez rarement fidèles. Ayant une trop forte personnalité, ils ne peuvent guère sortir d'eux-mêmes, échapper à leur univers pour entrer vraiment dans celui du poète qu'ils traduisent.

Il y a pourtant des exceptions. Un grand poète tel que Valéry a fourni des Bucoliques de Virgile une traduction en vers qui cst admirable par son exactitude, sa souplesse, sa beauté. Personnellement, je compte cette traduction parmi les meilleures de ses cuvres. Citons le début de la première Bucolique intitulée Tityre. C'est une conversation entre deux bergers, Tityre et Mélibée.

\section{Meliboeus -}

Tityre, tu patulae recumbans sub tegmine fagi

Silvestrum tenui musam meditaris avena;

Nos patriae fines et dulcia linquimus arva;

Nos patriam fugimus; tu, Tityre, lentus in umbra

Formosam resonare doces Amaryllida silvas.

Mélibée -

\section{Virgile}

O Tityre, tandis qu'à l'aise sous le hêtre

Tu cherches sur ta fiûte un petit air champêtre,

Nous, nous abandonnons le doux terroir natal,

Nous fuyons la patrie, et toi, tranquille à l'ombre,

Tu fais chanter au bois le nom d'Amaryllis. Paul Valéry

C'est très beau et très proche du texte latin, sans être d'une exactitude servile ${ }^{17}$.

17. P. Valéry, Eivves, Pléiade, I, p. 224, 225. R.A. Schröder a fourni, lui aussi, une Nachdichtung des Bucoliques de Virgile. Elie est fort belle aussi, mais moins dense, un peu redondante. Cf. Bucolica - Hirtengedichte, Suhrkamp Verlag, 1952 (édition magnifique, illustrée par Maillol). 
Examinons maintenant quelques difficultés majeures de la traduction poétique et essayons de dégager quelques règles.

La première question qui se pose lorsqu'on traduit un poème est celle de la versification (rythme et rime). La traduction se doit de respecter la disposition en strophes, la longueur des vers. On n'a pas le droit de traduire un vers court par un vers notablement plus long, car c'est une infidélité grave, inadmissible. On doit, si possible, rendre un vers allemand de 4 accents par un octosyllabe. Si à l'essai cela se révèle impossible, on peut aller jusqu'au décasyllabe, mais en aucun cas jusqu'à l'alexandrin. De même, le vers dramatique allemand (5 accents) doit se traduire, si possible, par un décasyllabe, à la rigueur par un alexandrin. On rendra l'Alexandriner allemand (6 accents), qui a été surtout en usage au XvTr ${ }^{e}$ siècle, par l'alexandrin français, et non par un vers de 14 syllabes. Si l'on choisit pour la traduction un vers plus long que celui de l'original, la tâche du traducteur en sera allégée; il ne se sentira pas à l'étroit; mais il aura tendance à allonger, à empâter. Le poème perdra de sa densité. Or, l'une des règles d'or de la poésie consiste à dire le plus de choses possible avec le moins de mots possible; on n'atteint l'intensité qu'à ce prix. Le traducteur tendra donc à faire aussi court, voire plus court que l'original, quitte à sacrifier des éléments secondaires, un peu de tissu conjonctif. C'est parfois possible, par ex. chez un écrivain un peu redondant comme Schiller : c'est alors un triomphe du traducteur. La traduction poétique est une excellente école de concision : elle donne l'habitude d'être sobre, d'économiser les mots et, lorsqu'on a le choix, de préférer le mot le plus court. Par ex., entre rocher, roche et roc, la préférence du traducteur ira probablement à roc, d'autant plus que le mot a la dureté et la rugosité qui conviennent au sens.

Voici un beau poème de Hofmannsthal, Reiselied, où la première strophe, un quatrain, énergique et rude, contraste avec la $2^{\mathrm{e}}$ et la $3^{\mathrm{e}}$ strophe, deux tercets d'une grande douceur.

\section{Reiselied}

Wasser stürzt, uns zu verschlingen,

Rollt der Fels, uns zu erschlagen,

Kommen schon auf starken Schwingen

Vögel her, uns fortzutragen.

Aber unten liegt ein Land.

Früchte spiegelnd ohne Ende

In den alterslosen Seen.

Marmorstein und Brunnenrand

Steigt aus blumigem Gelände,

Und die leichten Winde wehn.

Le traducteur a essayé de rendre ce contraste, de restituer la dureté de la $1^{\text {re }}$ strophe en utilisant des mots tels que torrent et roc et de multiplier les $\mathrm{r}$, comme dans le texte allemand; puis d'accumuler dans les tercets les sonorités douces : 
Chant du voyageur

Le torrent court pour nous noyer.

Le roc pour nous tuer dévale.

De grands oiseaux à tire-d'aile

Viennent déjà nous emporter.

Mais en bas s'étend un pays

Où de vieux lacs qui n'ont plus d'âge

Mirent les fruits à l'infini.

Marbres et fontaines surgissent

De ce paysage fleuri

Et sur le tout la brise glisse.

On peut constater que le traducteur a réussi à trouver des assonances et des rimes sans forcer le sens ${ }^{18}$. Le mètre a été respecté : le vers de 4 accents est transposé en octosyllabes; il n'y a donc pas une syllabe de plus.

Venons-en à la rime, problème que nous venons d'aborder. La rime est une difficulté presque infranchissable quand il s'agit de deux langues qui ne sont pas proches parentes comme le sont l'allemand et l'anglais, le français et l'italien. Quand un Allemand traduit un poème anglais, il lui est souvent possible de rimer sans déformer le sens. Quand un Anglais traduit de l'allemand, il en est de même. J'ai par ex. rencontré une traduction d'un sonnet de Rilke intitulé Eva et qui porte sur une statue d'Ėve située sur la façade de NotreDame de Paris. J'en cite deux strophes :

Einfach steht sie an der Kathedrale grossem Aufstieg, nah der Fensterrose, mit dem Apfel in der Apfelpose, schuldlos-schuldig ein für alle Male

an dem Wachsenden, das sie gebar, seit sie aus dem Kreis der Ewigkeiten

liebend fortging, um sich durchzustreiten durch die Erde, wie ein junges Jahr.

L'Anglais J.B. Leishman, un traducteur renommé ${ }^{19}$, a rendu ce sonnet avec une perfection incroyable. Le texte anglais, fidèle quoique très bien rimé, est aussi beau que l'original :

She, on the cathedral's vast ascent, simply stands there near the window-rose, with the apple in the apple-pose, ever henceforth guilty-innocent

of the growingness she brought to birth since that time she lovingly departed from the old eternities and started struggling like a young year through the Earth ${ }^{20}$.

18. Hugo von Hofmannsthal, Gedichte u. Lyrische Dramen, S. Fischer Verlag 1970, p. 11. La traduction (inédite) est de $A$. Schneider.

19. R.M. Rilke, Gesammelte Gedichte, Insel Verlag, 1962, p. 340, 341.

20. J.B. Leischman, "Betrachtungen eines englischen Rilke-Übersetzers", in : Die Kunst der Übersetzung, ouvrage collectif, déjà cité, p. 152, 153. 
Jaloux de cette réussite exceptionnelle, j'ai voulu rivaliser avec Leishman et j'ai traduit Eva à mon tour :

Très simple, elle est debout contre la cathédrale

- O grandiose montée! - auprès de la rosace,

Avec sa pomme, dans la pose de la pomme,

Innocente aussi bien coupable à jamais

Des développements qu'elle avait enfantés

Depuis le jour qu'aimante elle avait dû quitter

Le jardin éternel pour traverser le monde

En luttant pas à pas comme une jeune année.

Ma traducion ne vaut pas celle de mon collègue anglais. Je pense que ce n'est pas seulement dû à l'infirmité de mes moyens, mais aussi à la différence des langues : la transposition se fait beaucoup mieux en anglais qu'en français ${ }^{21}$.

Quand donc on a à traduire un poème, on a deux solutions : ou bien l'on rime, et l'on a toute chance de devenir infidèle afin de dénicher les rimes nécessaires; ou bien l'on ne rime pas, et l'on perd une bonne partie de la musique du vers. Les règles à suivre sont à mon avis les suivantes :

1) ne pas chercher systématiquement la rime; c'est trop périlleux ;

2) ne pas non plus fuir systématiquement la rime.

Ce sont là les règles suivies par Paul Valéry dans sa traduction en vers des Bucoliques de Virgile. Il ne rime pas, mais il ne repousse pas non plus la rime qui se présente. On a sans doute remarqué dans les paroles de Mélibée que j'ai citées les rimes hêtre, champêtre. Maurice Boucher, dans ses traductions de Stefan George, procède de même. Certaines traductions de George ne sont pas rimées, par ex. celle de Brise marine de Mallarmé, qui est à la fois belle et remarquablement exacte ${ }^{22}$.

$\mathrm{La}$ rime peut se remplacer par une musique plus subtile, moins éclatante, moins perceptible, mais cependant sensible : assonances, allitérations, rimes intérieures, harmonie des voyelles et des consonnes. Un poème peut être très musical sans être rimé. Tous les vrais amateurs de poésie le savent. Cependant, le miracle se produit parfois : des rimes se présentent presque d'elles-mêmes; on peut quelquefois, sans solliciter le texte, rimer tout un poème. J'ai pu le faire pour un poème de Lenau intitulé Melancholie ${ }^{23}$. Un bon exemple d'une traduction rimée est la transposition de la Carpe d'Apollinaire par Karl Krolow, grand poète et grand traducteur.

Dans vos viviers, dans vos étangs,

Carpes, que vous vivez longtemps!

Est-ce que la mort vous oublie,

Poissons de la mélancolie.

\section{Apollinaire}

21. On trouvera une autre traduction d'Eva, par Jacques Legrand, dans R.M. Rilke. Euvres, 2, Poésie, Ed. Seuil, Paris, 1972, p. 249. Elle est en vers irréguliers.

22. Citée par Manfred Gsteiger, Französische Symbolisten in der deutschen Literatur der Jahrhundertwende, Francke, Bern, 1971, p. 178.

23. Lenau, Werke, Bibliograph. Institut, Leipz. u. Wien, 1910, I, 146. Traduction de A. Schneider dans la Nouvelle Revue Luxembourgeoise, 1972, no 1, p. 43 . 
In euren Teichen, euren Gräben :

Wie mögt ihr, Karpfen, lange leben !

Holt euch der Tod am Ende nie,

Geschöpfe der Melancholie?

\section{Karl Krolow}

Cette traduction rend parfaitement l'atmosphère du quatrain et le rythme des vers ${ }^{24}$. Elle est à la fois rimée et fidèle, bien que sans servilité.

Il est rare que l'auteur d'une traduction poétique soit pleinement satisfait de son travail. Un bon traducteur - comme beaucoup de poètes qui remanient volontiers leurs textes, par ex. Hölderlin, C.F. Meyer, Trakl - fait, défait et refait sans cesse ses traductions. Une transposition de poème n'est jamais terminée. L'auteur s'arrête à une certaine solution, c'est tout. Cette solution, ce n'est à peu près jamais la première. De tous les poèmes que j'ai traduits, il existe 2 ou 3 versions, parfois davantage, en dehors de la première version en prose, quand elle existe.

Quelles sont les différences entre les versions successives du même texte? Souvent, il n'y a qu'une différence de niveau. Les solutions apportées aux difficultés deviennent plus précises, plus satisfaisantes, plus élégantes. Parfois, il n'y a entre la $2^{\mathrm{e}}$ et la $3^{\mathrm{e}}$ version que 2 ou 3 vers modifiés, que quelques mots de changés. D'autres fois, les différences sont notables. Une version est en vers blancs, l'autre est rimée. Plus souvent, une version est dans un certain mètre, mettons en décasyllabes; la suivante sera en octosyllabes ou en alexandrins, suivant les cas.

Quand on passe de l'alexandrin au décasyllabe, ou du décasyllabe à l'octosyllabe, cela pose de difficiles problèmes. Le traducteur doit faire un gros effort de concision. Donc, si le mètre change, le texte changera aussi, forcément. Donnons un exemple. Clemens Brentano, poète romantique à la poésie rêveuse, musicale, fluide, a écrit un ravissant poème intitulé Abendständchen :

Hör, es klagt die Flöte wieder,

Und die kühlen Brunnen rauschen,

Golden wehn die Töne nieder -

Stille, stille, lass uns lauschen !

Holdes Bitten, mild Verlangen,

Wie es süss zum Herzen spricht!

Durch die Nacht, die mich umfangen,

Blickt zu mir der Töne Licht.

Ce sont des vers trochaïques de 4 pieds, donc en principe de 8 syllabes. Me donnant un peu de jeu, j'ai d'abord essayé de les transposer en décasyllabes et de rendre la douceur des sons et la fluidité du rythme, tout en respectant le sens, bien entendu :

24. Apollinaire, Euvres poétiques, Ed. Pléiade, Gallimard, Paris, 1965. Le Bestiaire, p. 25. La traduction est citée et commentée par $K$. Krolow lui-même dans un article sur «Der Lyriker als Übersetzer zeitgenössischer Lyrik», in : Die Kunst der Übersetzung, déjà cité, p. 124. 


\author{
Sérénade
}

Écoute gémir à nouveau la flûte

Et murmurer les fontaines glacées,

Déversant par nuages des sons d'or.

Silence, ô silence ! Prêtons l'oreille!

Prières tendres, douce nostalgie,

Quel caressant langage pour le cour !

Trouant la nuit qui m'entoure, les sons

Braquent sur moi leur regard de lumière.

Dès le lendemain, bien que relativement content de mon travail, je n'ai pu résister au plaisir peut-être un peu pervers d'accroître encore la difficulté et j'ai composé une nouvelle version en octosyllabes. Là, je me suis enfermé dans un rude corset, car dans une telle traduction, chaque syllabe compte. Si l'on en ajoute une seul'e, rien ne va plus; si l'on change un seul mot, presque tout est à recommencer, car un poème est un être vivant, un organisme où tout est lié. Jean Cocteau a insisté à juste titre sur cette idée. J'ai cssayé de préserver le sens, la musique des voyelles et des consonnes, le glissando du rythme. On notera que le texte, par rapport à la première version, est presque entièrement renouvelé, et pourtant, tout $\mathrm{y}$ est :

Écoute! À nouveau geint la flûte

Et jasent les fontaines fraîches.

Comme d'or dévalent les sons -

Ne parlons plus! Prêtons l'oreille !

Tendres prières, doux désirs,

Un charme s'insinue au cour.

Trouant la nuit qui m'enveloppe

Les sons m'adressent leurs lueurs.

L'auteur se gardera de dire quelle version il préfère : il est juge et partie ${ }^{25}$.

Notre sujet est loin d'être épuisé. Mais il est temps de conclure.

Même pour un traducteur doué et expérimenté, même pour un poète, la traduction poétique est et reste une entreprise audacieuse, hasardeuse. Les difficultés sont telles que le calcul des probabilités - et les savantes recherches des linguistes - sont contre les «translateurs » de poèmes. Mais l'on constate aussi qu'il se trouve encore et toujours des traducteurs pour affronter cette tâche ingrate, mais noble, et parmi eux, il y a beaucoup de poètes, de grands poètes. Après tout, la poésie elle-même est une entreprise tout aussi utopique, tout aussi hasardeuse que la traduction poétique, et pourtant, des millénaires n'ont pas mis fin à l'activité des poètes. Au siècle où nous sommes, siècle de la raison, de la science et de la technique, la poésie, très transformée, il est vrai, poursuit allègrement son chemin.

Elsa Triolet, la compagne d'Aragon, a écrit dans la préface à une $A n$ thologie bilingue de la poésie russe parue en $1965 \mathrm{chez}$ Seghers que la traduc-

25. Clemens Brentano, Gedichte, Insel-Bücherei Nr. 117, p. 33. Traductions inédites de A.
Schneider. 
tion poétique est un «travail pénible, épuisant, irritant». Ce n'est pas notre sentiment. C'est un travail difficile, certes, mais passionnant et très enrichissant. Outre qu'il met à la portée des lecteurs la poésie des nations étrangères, il apporte au traducteur les enseignements les plus divers.

Tout d'abord, le traducteur apprend à se connaître lui-même, à mesurer et à mobiliser ses forces et ses possibilités et à les accroître systématiquement par l'exercice, ce mot étant toujours pris au sens valéryen. L'exercice lui apprend à affronter les difficultés, à vaincre les obstacles - ou du moins à les reconnaître clairement et avec précision. Il apprend à reconnaître la nature des poètes, à se rendre compte de leurs points communs, de leurs divergences. Il apprend à reconnaître la nature du langage poétique, avec ses différents aspects, ses variations, son évolution. Il apprend à reconnaître le génie de deux langues, la langue de départ et la langue cible, la différence de leur organisation, de leur orientation, de leurs ressources, de leur évolution. La traduction poétique apprend enfin au traducteur, mieux que toute poétologie, toujours trop générale et trop abstraite, à reconnaître concrètement la nature de la poésie, la façon subtile et un peu mystérieuse dont elle naît, les éléments qui la font surgir et auxquels elle est liée, ainsi que ceux qui la dissipent et la chassent.

Par conséquent, même si un traducteur de poèmes n'a à son actif qu'un petit nombre de pleines réussites, il n'a pas lieu d'être déçu et de se plaindre. De même que le poète est récompensé par son poème, le traducteur trouve joie et récompense dans sa traduction. On peut lui appliquer le beau vers qui termine le poème de Mörike, Auf eine Lampe :

Was aber schön ist, selig scheint es in ihm selbst.

Ce que l'auteur de ces pages a traduit comme suit :

Ce qui vraiment est beau mûrit en soi l'extase ${ }^{26}$.

Albert SCHNeIDER

26. Traduction parue dans la Nouvelle Revue Luxembourgeoise $1972, \mathrm{n}^{\circ} 1$, p. 44. 